\title{
Phosphorylated mTOR and YAP serve as prognostic markers and therapeutic targets in gliomas
}

\author{
Mei Liu'1,2,3, Yong Lin 1,2,3, Xian-Chao Zhang ${ }^{1,2}$, Yu-Huan Tan ${ }^{1,2}$, Yue-Liang Yao ${ }^{1,2}$, Juan Tan ${ }^{1,2}$, Xia Zhang ${ }^{1,2}$, \\ You-Hong Cui ${ }^{1,2}$, Xindong Liu ${ }^{1,2}$, Yan Wang ${ }^{1,2}$ and Xiu-Wu Bian ${ }^{1,2}$
}

Glioma is the most prevalent type of tumor in the brain and is comprised of grades I-IV, according to the WHO classification system. Grade IV glioma is also known as glioblastoma multiforme (GBM), the most malignant type of glioma. Glioma is characterized by a complex molecular background, and gene profiling studies have disclosed critical genetic events in human gliomas, which make targeted therapies the most promising therapeutic strategy. However, crosstalk between the targeted signaling pathways may hinder the efficacy of targeted therapies in gliomas. Therefore, it is necessary to identify effective markers to stratify patients for specific therapeutic procedures. Although several mechanisms have been proposed based on the crosstalk between PI3K/AKT/mTORC1 and Hippo/YAP pathways, the clinical significance of the two pathways has not yet been assessed in a combinatorial manner. In this study, we evaluated the two pathways in human glioma specimens and observed the positive correlation between protein levels of p-mTORS2448 and YAP in gliomas. The findings indicated that high expression of p-mTORS2448 and YAP correlated with poor overall survival of glioma patients. As p-mTORS2448 is a specific marker of mTORC1 activation, our results reveal a potential interaction between mTORC1 and YAP, which might functionally participate in the development and progression of gliomas. In support of this hypothesis, a combination of inhibitors targeting mTORC1 and YAP showed a better inhibitory effect on growth of glioma cell lines. Altogether, our work, for the first time, reveals that p-mTORS2448 and YAP can be used as markers of PI3K/AKT/mTORC1 and Hippo/YAP pathway activity to predict prognosis and are target candidates for personalized medicine.

Laboratory Investigation (2017) 97, 1354-1363; doi:10.1038/labinvest.2017.70; published online 31 July 2017

Glioma is the most prevalent malignancy in the brain and has been characterized as having a complex molecular background. ${ }^{1}$ Based on the WHO classification method, gliomas are divided into four grades: I, II, III, and IV. Grade IV glioma is also known as glioblastoma (GBM) (primary GBM), and grade III glioma has high potency to progress to GBM (secondary GBM). ${ }^{2}$ Despite the progress in surgical, radiotherapy, and/or chemotherapy, the primary and secondary GBMs are still intractable and show poor prognoses. ${ }^{3,4}$ Because gene profiling studies have unveiled critical genetic events in human gliomas, target therapies are emerging as promising strategy of precision medicine. However, the complexity of signaling pathways related to these genetic events results in uncertainty as to what would be the appropriate targets and confines the application of target therapies in gliomas. ${ }^{5,6}$ Therefore, it is necessary to identify effective markers to stratify patients for specific target therapies.

In gliomas, two development-related signaling pathwaysHippo and $\mathrm{mTOR}^{7-10}$ — have been investigated, respectively, and are involved in the development and progression of gliomas. ${ }^{11,12}$ The Hippo pathway is highly conserved from drosophila to humans ${ }^{13,14}$ and includes a group of tumor suppressors, such as Merlin, mammalian STE-20 kinase (Mst), WW-domain protein (WW45), large tumor suppressor (Lats), and Msp-one-binder 1 (Mob1). The major target of the Hippo pathway is Yes-associated protein (YAP) and transcriptional coactivator with PDZ-binding motif (TAZ, also known as WWTR1) (YAP/TAZ), which functions as a transcriptional factor to promote cell proliferation and inhibit

\footnotetext{
${ }^{1}$ Department of Pathology, Institute of Pathology and Southwest Cancer Center, Southwest Hospital, Third Military Medical University, Chongqing, China and ${ }^{2}$ Key Laboratory of Tumor Immunology and Pathology of Ministry of Education, Chongqing, China.

Correspondence: Y Wang, PhD or X-W Bian, PhD, MD, Department of Pathology, Institute of Pathology and Southwest Cancer Center, Southwest Hospital, Third Military Medical University, Chongqing 400038, China.

E-mail: wang_yan1977@hotmail.com or bianxiuwu@263.net

${ }^{3}$ These authors contributed equally to this work.

Received 9 February 2017; revised 28 April 2017; accepted 26 May 2017
} 
apoptosis. ${ }^{15-17}$ In general, when cells are under adverse conditions, eg, high cell density and low nutrition, the Hippo pathway is activated through the stimulation of the tumor suppressors, which in turn phosphorylate YAP/TAZ and retains the complex in cytosol without transcriptional activity. ${ }^{18-20}$ However, when Hippo pathway switches off, non-phosphorylated YAP/TAZ is accumulated in the nucleus and is functionally activated. ${ }^{21,22}$ The aberrant activation of $\mathrm{YAP} / \mathrm{TAZ}$ is frequently detected in cancers and correlates with poor prognosis, ${ }^{23-26}$ and YAP/TAZ has been considered as a drug target to antagonize YAP/TAZ-related cancers. ${ }^{27,28}$

The mechanistic target of rapamycin (mTOR) signaling pathway has an important role in many aspects of cell behavior, such as proliferation, angiogenesis, cell survival, and metabolism. ${ }^{29}$ mTOR forms two complexes with different partners, mTOR complex 1 (mTORC1) and mTOR complex 2. ${ }^{29,30}$ Raptor is essential for the efficient phosphorylation of mTORC1 downstream targets, whereas Rictor is critical for mTORC2 function. mTORC1 is activated by PI3K/AKT axis ${ }^{31}$ through phosphorylation of mTOR Ser $2448^{32,33}$ and activated mTOR phosphorylates p70S6K and 4E-BP1, leading to an increase of the protein translation rate. ${ }^{34}$ Dysregulation of the pathway has been found to contribute to various types of cancer, ${ }^{35,36}$ and several inhibitors of this pathway have been tested in clinical trials. ${ }^{36}$ Moreover, PI3K/AKT/mTOR signaling pathway is highly activated and potential target for target therapy in GBM. 37,38

Recent reports have provided evidence of links between the mTOR and Hippo/YAP pathways, and the crosstalk of the two pathways regulates tissue homeostasis ${ }^{10,39}$ and tumorigenesis. ${ }^{11,40}$ Previous studies have linked mTOR and Hippo/YAP. ${ }^{41,42}$ Specifically, mTORC1 engages in crosstalk with YAP through various mechanisms: YAP mediates crosstalk between the Hippo and PI3K-mTOR pathways by suppressing PTEN via miR-29 ${ }^{10}$ and YAP/TAZ enhances mTORC1 activity via increase of amino-acid uptake. ${ }^{39,43}$ Nevertheless, whether the association between YAP/TAZ and mTORC1 has clinical significance in human gliomas remains to be elucidated. In this study, we evaluated the expression of mTOR, p-mTORS2448 (mTORC1 specific phosphorylation form of mTOR), YAP, and p-YAP in clinical glioma specimens and found that both p-mTORS2448 and YAP were negatively correlated with prognosis of gliomas and simultaneous inhibition of both signaling pathways exerted more effective inhibition on the growth of glioma cells, which might provide a novel set of markers to stratify patients for appropriate combination therapy.

\section{MATERIALS AND METHODS Patient Samples}

In this study, all the glioma tissue samples were collected from Department of Pathology of Southwest Hospital, Third Military Medical University (TMMU) between November 2013 and June 2014. None of the patients had received radiotherapy or chemotherapy before surgery. All the tissue samples were immediately processed after surgical removal, fixed with $4 \%$ formalin ( $\mathrm{pH} \mathrm{7.0)}$ ), and embedded in paraffin for no $>24 \mathrm{~h}$. Pathological examination confirmed the diagnosis of glioma and was classified as glioma grade I-IV according to World Health Organization (WHO) criteria. The complete clinical and prognostic data for each tumor tissue sample were recorded. The survival time was calculated from the date of disease confirmed to death, and the dates of death were obtained from the public records. All protocols have been approved by the ethics committee of Southwest Hospital of TMMU.

\section{Cell Culture}

The human embryonic brain (HEB) cell line was kindly provided by Dr Guangmei Yan (Zhongshan School of Medicine, Sun Yat-Sen University, China). $\mathrm{CHG}^{44}$ and SHG $44^{45}$ cells were established from the tumor specimens of patients with glioma (Southwest Hospital, TMMU). LN229 (human GBM cell line) was a kind gift from the College of Pharmacy at our university. T98G (human GBM cell line) was purchased from American Type Culture Collection (ATCC). All the cells were maintained in Dulbecco's Modified Eagle Medium (Gibco) containing 10\% FBS (Gibco) and incubated at $37^{\circ} \mathrm{C}$ in a humidified incubator with $5 \% \mathrm{CO}_{2} / 95 \%$ air.

\section{Immunohistochemistry (IHC)}

Surgical specimens were obtained from patients with gliomas (Southwest Hospital, TMMU). The follow protocol for IHC was performed according to our previous study. ${ }^{46}$ Primary antibodies that included anti-YAP antibodies (D24E4, rabbit monoclonal), anti-p-YAP antibodies (Ser127, rabbit monoclonal), anti-p-mTORS2448 antibodies (D9C2, rabbit monoclonal), and anti-mTOR antibodies (7C10, rabbit monoclonal) were purchased from Cell Signaling Technology. On each slide, a positive control for each antibody tested was included. The evaluation of IHC staining as follows: Five most characteristic high-power fields $(\times 400$ magnification $)$ per tissue section were manually selected using an Olympus BX51 microscope (Olympus, Tokyo, Japan). The respective areas of tumor islets and stromal were defined and measured using the Image-Pro Plus 6.0 software (Media Cybernetics, Silver Spring, MD, USA). As a substitute for the traditional grading method, IHC results that represent protein expression level were analyzed by Image-Pro Plus software to acquire accurate value of integral optic density and area in order to calculate average optical density. ${ }^{47}$

\section{IC50 Calculation}

Cells were seeded in 96-well plates at a density of 3000 cells/well. After overnight growth, the cells were exposed to Everolimus and peptide 17 with increasing concentrations, ranging from 0 and $80 \mu \mathrm{M}$ for $48 \mathrm{~h}$. The concentrations required to inhibit cell growth by $50 \%$ (IC50) were calculated from survival curves. 


\section{Cell Proliferation Assay}

HEB and tumor cells (CHG5, SHG44, T98G, and LN229) were seeded into 96-well plates and treated for 24, 48, 72, and $96 \mathrm{~h}^{28,48}$ using Everolimus and peptide 17 alone with IC50 concentrations we have measured (Figure 5a) or in combination with half of IC50 concentrations for each inhibitor, and DMSO was used as a control (drug vehicle). At the indicated intervals, $10 \mu \mathrm{l}$ of Cell Counting Kit-8 (Beyotime, China) were added to each well and incubated for $1 \mathrm{~h}$ at $37^{\circ} \mathrm{C}$. Thermo Multiskan Spectrum Reader (Thermo Scientific) was used to measure the absorbance at $450 \mathrm{~nm}$.

\section{Western Blotting}

HEB and tumor cells (CHG5, SHG44, T98G, and LN229) were treated for $12 \mathrm{~h}$ using Everolimus and peptide 17 alone with IC50 concentrations we have measured (Figure 5a) or in combination with half of IC50 concentrations for each inhibitor, and DMSO was used as a control (drug vehicle). Western blotting procedure was followed as previously described. ${ }^{49}$ All the antibodies were purchased from Cell Signaling Technology. The primary antibodies were as follows: anti-YAP (D24E4, rabbit monoclonal), anti-p-YAP (Ser127, rabbit monoclonal), anti-p-mTOR (Ser2448, 49F9, rabbit monoclonal), anti-mTOR (rabbit monoclonal), anti-pS6 (Ser235/236, rabbit monoclonal), and anti- $\beta$-actin (mouse monoclonal).

\section{Statistical Analysis}

Multiple statistical methods were used to summarize the results of our study participants. Significance was established with the Graphpad Prism 5 software (Graphpad Software, Inc, San Diego, CA, USA) and SPSS statistical package for Windows version 13 (SPSS, Chicago, IL, USA), using Pearson's Chi-squared test and Student's $t$-test when appropriate. Pearson's Chi-squared test was used to determine the correlation between YAP and p-mTORS2448 expression and clinical and pathological features of patients. Patients' survival was analyzed with Kaplan-Meier method, using the log-rank test for comparison. The statistical software X-tile was used to determine the cutoff in the glioma cohort 50. The correlation between the expression levels of different proteins was measured using nonparametric correlation test and curvilinear regression model. All experiments were performed at least three times with triplicate samples.

\section{RESULTS}

\section{Clinical-Pathological Characteristics of Patients}

Clinical-pathological information of 72 patients was summarized in Table 1. The patients' ages range from 4 to 72 years and included 38 males and 34 females. The gliomas were classified into four grades (I, II, III, and IV) by WHO classification. The grade I and II gliomas were generally referred to as low-grade gliomas (LGG), whereas the grade III and IV gliomas as high-grade gliomas (HGG) ${ }^{50}$ Tumor locations of the patients were varied but had no grade-specific
Table 1 Clinical features of the glioma specimens

\begin{tabular}{lcc}
\hline \multirow{2}{*}{ Feature } & LGG $(I+I I, n=32) \quad$ HGG $(\mathrm{III}+\mathrm{IV}, n=40)$ \\
\cline { 2 - 3 } & \multicolumn{2}{c}{ No. (\%) } \\
\hline $\begin{array}{l}\text { Gender } \\
\text { Male }\end{array}$ & $12(37.5)$ & $26(65)$ \\
Female & $20(62.5)$ & $14(35)$ \\
Age at diagnosis (year, mean \pm s.d.) & $34.2 \pm 1.62$ & $53.1 \pm 6.53$ \\
& & \\
Predominant side of tumor location & & $14(35)$ \\
Left & $14(43.7)$ & $25(62.5)$ \\
Right & $11(34.4)$ & $0(0)$ \\
Middle & $0(0)$ & $1(2.5)$ \\
$\quad$ Other & $7(21.9)$ &
\end{tabular}

Predominant lobe of tumor location

$\begin{array}{lcc}\text { Frontal } & 11(34.4) & 10(25) \\ \text { Temporal } & 7(21.9) & 16(40) \\ \text { Parietal } & 1(3.1) & 7(17.5) \\ \text { Occipital } & 1(3.1) & 2(5) \\ \text { Other } & 12(37.5) & 5(12.5)\end{array}$

First presenting symptom

Headache

Mental status change

$16(50)$

$20(50)$

Motor or movement change

$2(6.3)$

17 (42.5)

$14(43.7)$

$3(7.5)$

Postoperation therapies

Chemical therapy only

$10(25)$

$22(68.8)$

Radiotherapy and

$19(47.5)$

$17(53.1)$

chemotherapy

$\begin{array}{lrr}\text { IDH1 status } & & \\ \text { Mutant } & 17(53.1) & 9(22.5) \\ \text { WT } & 15(46.9) & 31(77.5)\end{array}$

$\begin{array}{lcc}\text { IDH2 status } & & \\ \text { Mutant } & 3(9.4) & 3(7.5) \\ \text { WT } & 29(90.6) & 37(92.5)\end{array}$

Abbreviations: HGG, high-grade glioma; LGG, low-grade glioma.

distributions. All the patients received chemotherapy or chemo-radiotherapy after surgical resection. In addition, both IDH1 and IDH2 mutations were identified in samples with total higher mutation rate in LGG than in HGG (53.1 vs $22.5 \%$ for IDH1 and 9.4 vs $7.5 \%$ for IDH2). 
a mTOR p-mTORS 2448

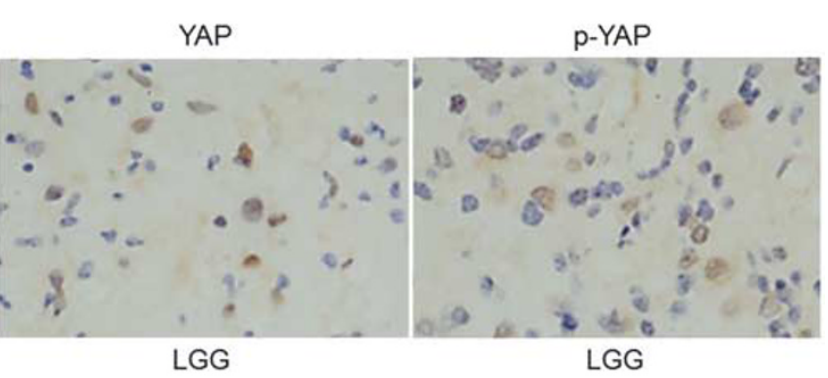

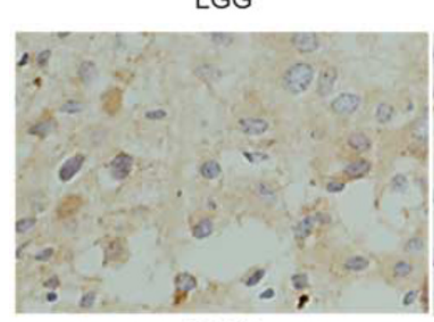

HGG

b $\quad \mathrm{mTOR}$

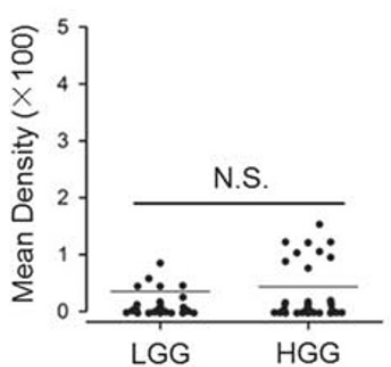

C
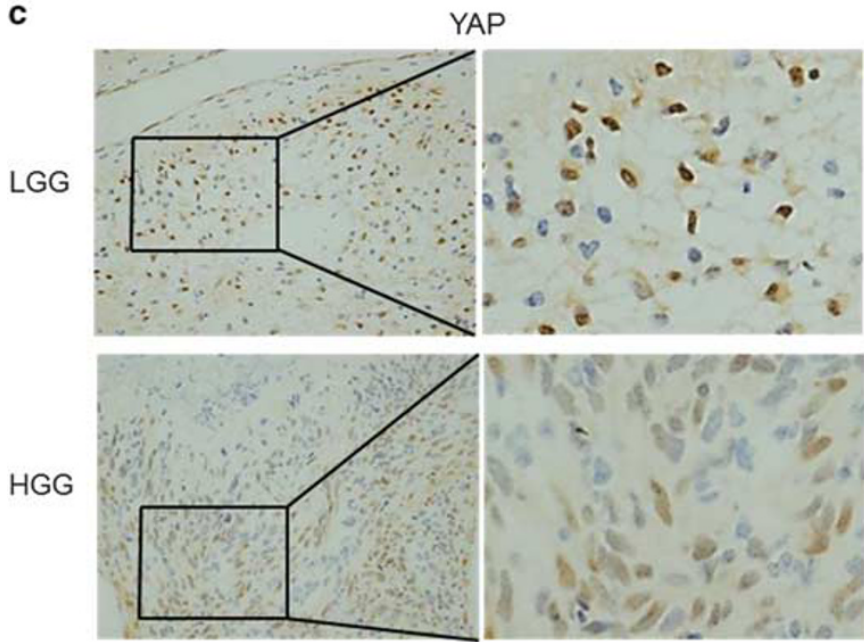

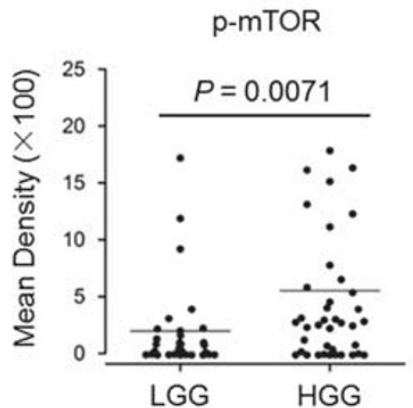

YAP
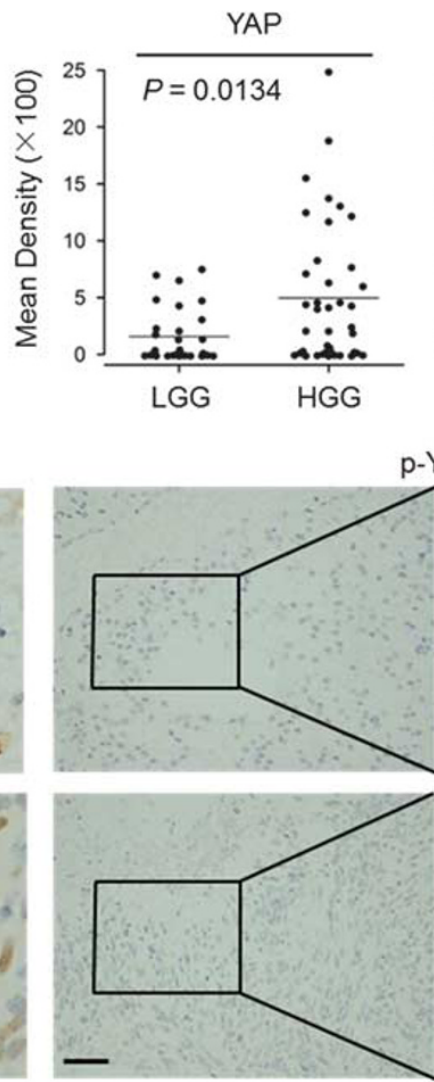

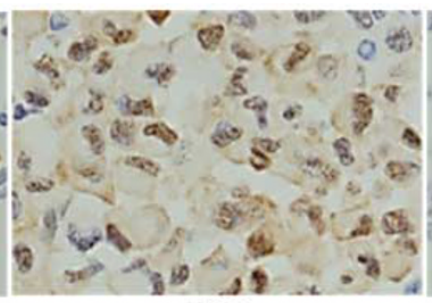

HGG
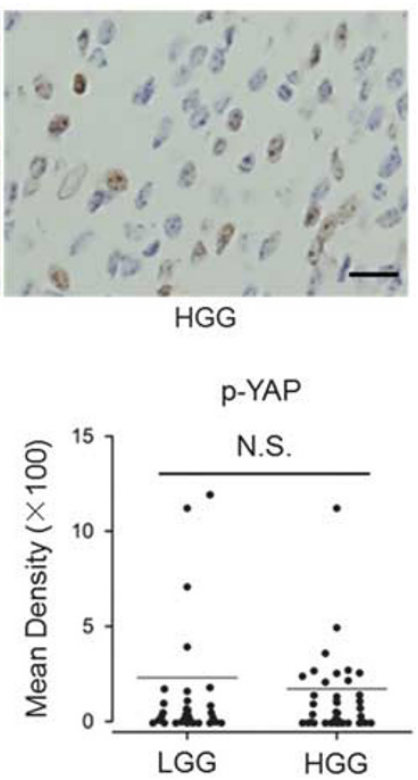

p-YAP
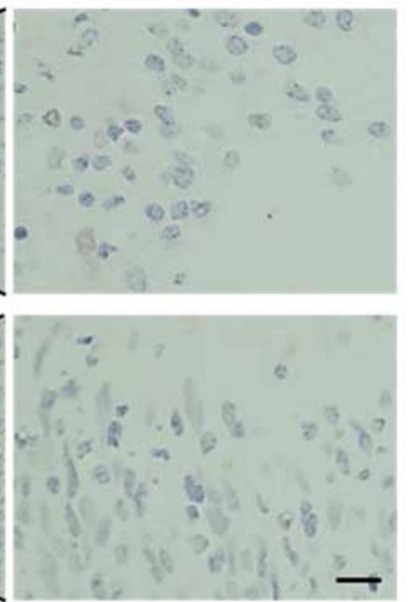

Figure 1 Expression of mTOR, p-mTORS2448, YAP and p-YAP in glioma tissues. (a) Representative images of glioma sections immunohistochemically stained for mTOR, p-mTORS2448, YAP and p-YAP in low-grade and high-grade gliomas (LGG and HGG, respectively). Scale bar $=25 \mu \mathrm{m}$. (b) Statistical analysis of staining results in LGG vs HGG and HGGs. Expression level was measured by the Image-Pro Plus 6.0 software. Student's $t$-test was used to calculate the $P$-value. NS means no statistical significance. (c) Continuous sections stained with YAP and p-YAP. Scale bar $=50 \mu \mathrm{m}$ (for original images) or $25 \mu \mathrm{m}$ (for insets).

\section{Protein Expression of mTOR, p-mTORS2448, YAP, and p-YAP in Gliomas}

To study the protein expression and status of mTOR and YAP in glioma tissues, we performed immunostaining with
anti-mTOR, anti-p-mTORS2448, anti-YAP, and anti-p-YAP antibodies (Figure 1a and Table 2). We detected high expression of mTOR in 19 of LGG $(59.45 \%)$ and 22 of HGG (55\%), p-mTORS2448 in 11 of LGG (34.4\%) and 31 of 
HGG (62.5\%), YAP in 20 of LGG (62.5\%) and 25 of HGG (77.5\%), and p-YAP in 22 of LGG $(68.7 \%)$ and 23 of HGG $(57.5 \%)$ (Table 2). Statistical analysis indicated that p-mTORS2448 and YAP were significantly elevated in HGG vs LGG $(P=0.0071$ for $\mathrm{p}$-mTORS2448 and $P=0.0134$ for YAP), but not in mTOR and p-YAP (Figure 1b). It is well known that YAP functions as a transcriptional factor, and its nuclear localization represents its activation. Our data indicate that high YAP staining was also accompanied by strong nuclear staining (Figure 1c), which suggests that the YAP protein level is a reliable indicator for YAP activity in gliomas. It was noted that the IHC staining indicated that not all cells evenly expressed these proteins (Figures 1a and c),

Table 2 Staining results of different antibodies on the glioma specimens

\begin{tabular}{lccc}
\hline Antibody & $\begin{array}{c}\text { Low expression, } \\
\text { no. (\%) }\end{array}$ & $\begin{array}{c}\text { High expression, } \\
\text { no. (\%) }\end{array}$ & $\begin{array}{c}\text { Total stained and } \\
\text { interpreted, no. (\%) }\end{array}$ \\
\hline $\begin{array}{l}\text { mTOR } \\
\text { LGG }\end{array}$ & $13(40.6)$ & $19(59.4)$ & $32(100)$ \\
HGG & $18(45)$ & $22(55)$ & $40(100)$ \\
p-mTORS2448 & & & \\
LGG & $21(65.6)$ & $11(34.4)$ & $32(100)$ \\
HGG & $15(37.5)$ & $25(62.5)$ & $40(100)$ \\
YAP & & & $32(100)$ \\
LGG & $12(37.5)$ & $20(62.5)$ & $40(100)$ \\
HGG & $9(22.5)$ & $31(77.5)$ & \\
& & & $32(100)$ \\
p-YAP & $10(31.3)$ & $22(68.7)$ & $40(100)$ \\
LGG & $17(42.5)$ & $23(57.5)$ & \\
HGG & & & \\
\hline
\end{tabular}

which might result from the heterogenicity of glioma that has been well documented and is thought to be a critical factor when considering treatment responses for glioma. ${ }^{1,4}$

\section{Correlation of Expression of p-mTORS2448 and YAP in Gliomas}

As only p-mTORS2448 and YAP were expressed significantly differently between HGG vs LGG, we further evaluated the expression between the two proteins. Pearson's analysis revealed a previously unreported result that the expression p-mTORS2448 and YAP are positively correlated with each other (Figure 2a). IHC on continuous tissue sections further confirmed that high expression of p-mTORS2448 and YAP were concurrent in the same samples (Figure 2b), implying that a crosstalk between mTOR and Hippo pathways might exist in gliomas through the coordination of p-mTORS2448 and YAP.

\section{Relationship Between p-mTORS2448/YAP Expression and IDH Status in Gliomas}

IDH1 and IDH2 are considered important prognostic markers for glioma patients, and mutated IDH is often related to improved prognosis. Thus we asked whether the expression of p-mTORS2448 and YAP was also related with the IDH status in glioma. In our cohort, there was no statistically significant difference in the expression of p-mTORS2448 or YAP in glioma samples with IDH mutation $v s$ those with IDH wild type (Figure 3), which implies that the mTOR and YAP pathways are independent of the IDH status in glioma.

\section{Prognostic Significance of p-mTOR and YAP in Gliomas}

Using Kaplan-Meier survival analysis, we found that both p-mTORS2448 and YAP overexpression were associated with lower overall survival rates in all the patients $(P<0.0001$ and $P=0.0002$, respectively; Figures $4 \mathrm{a}$ and $\mathrm{b}$ ). Moreover, the combination of expression of p-mTORS2448 and YAP was also negatively related with the overall survival of patients $(P=0.0052$; Figure $4 \mathrm{c})$. As p-mTORS2448 and YAP were

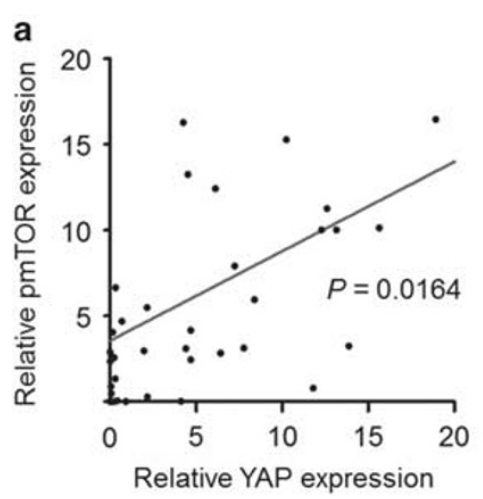

b

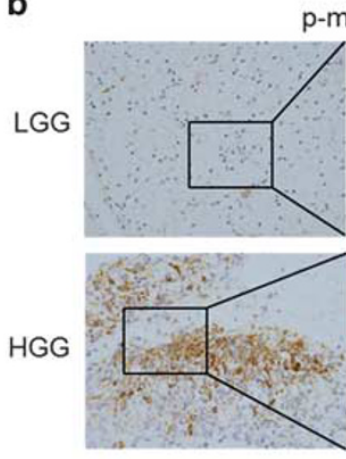

-mTOR

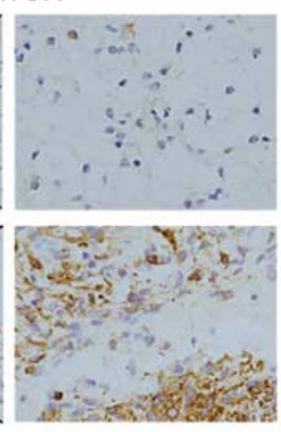

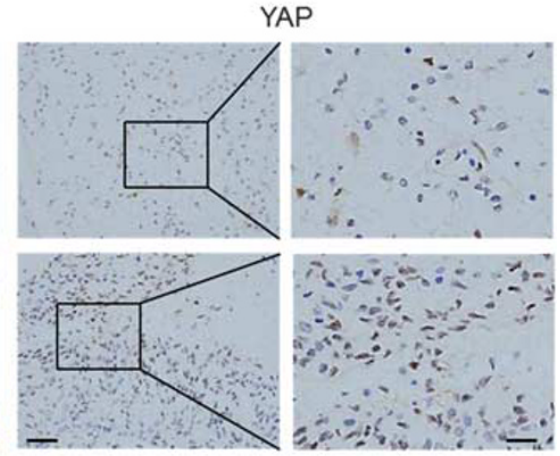

Figure 2 Correlation of p-mTORS2448 and YAP in glioma tissues. (a) Correlation of p-mTOR and YAP via Pearson analysis in glioma tissues. (b) Representative immunohistochemical staining for $\mathrm{p}$-mTOR and YAP on the continuous glioma specimens. Scale bar $=50 \mu \mathrm{m}$ (for original images) or $25 \mu \mathrm{m}$ (for insets). 

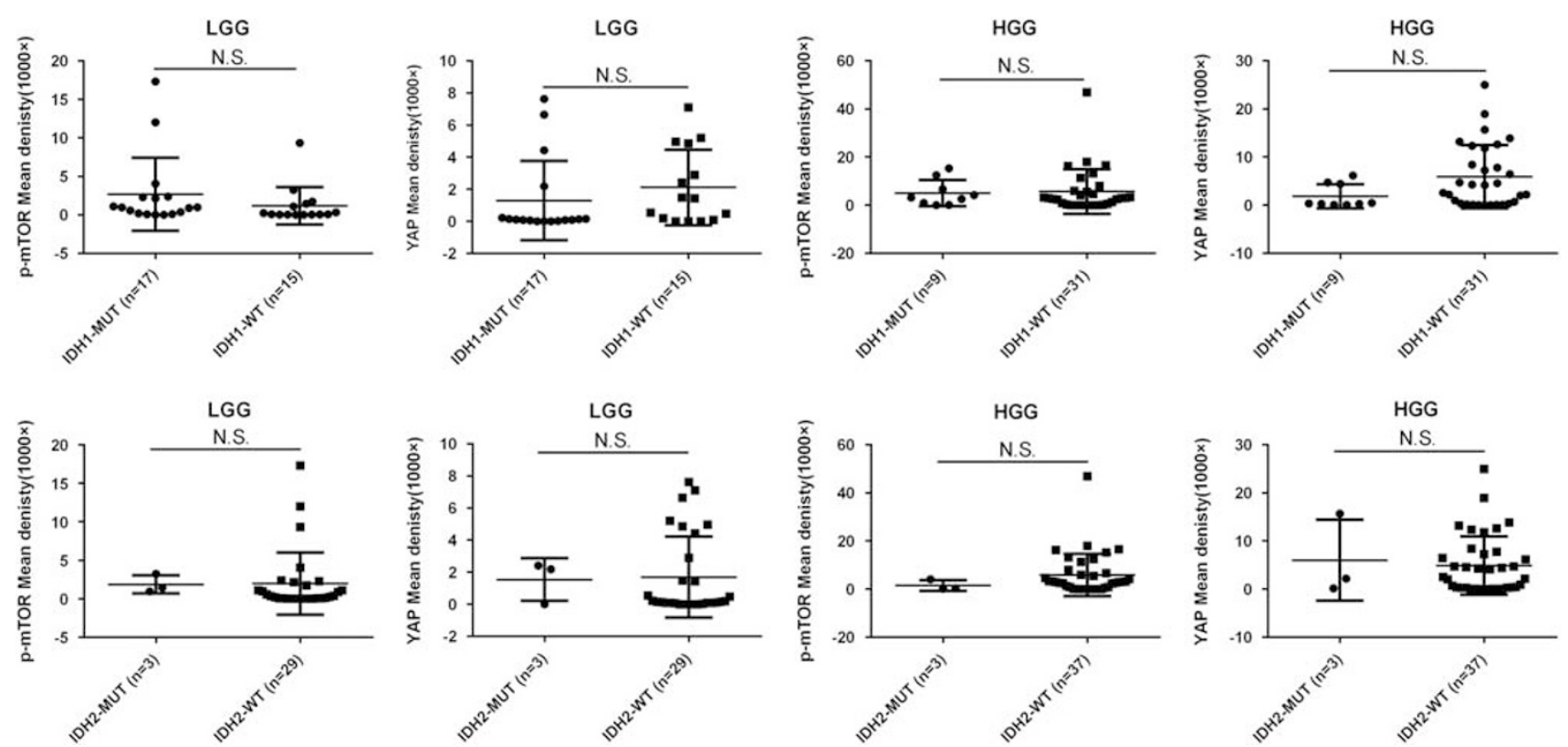

Figure 3 The expression of p-mTORS2448 and total YAP in glioma samples with IDH mutant vs wild type. Upper four panels and lower four panels represent the expression of the two proteins in glioma samples with IDH1 mutant vs wild type and IDH2 mutant vs wild type, respectively. Student's $t$-test is used to calculate the $P$-value. NS means no statistical significance.
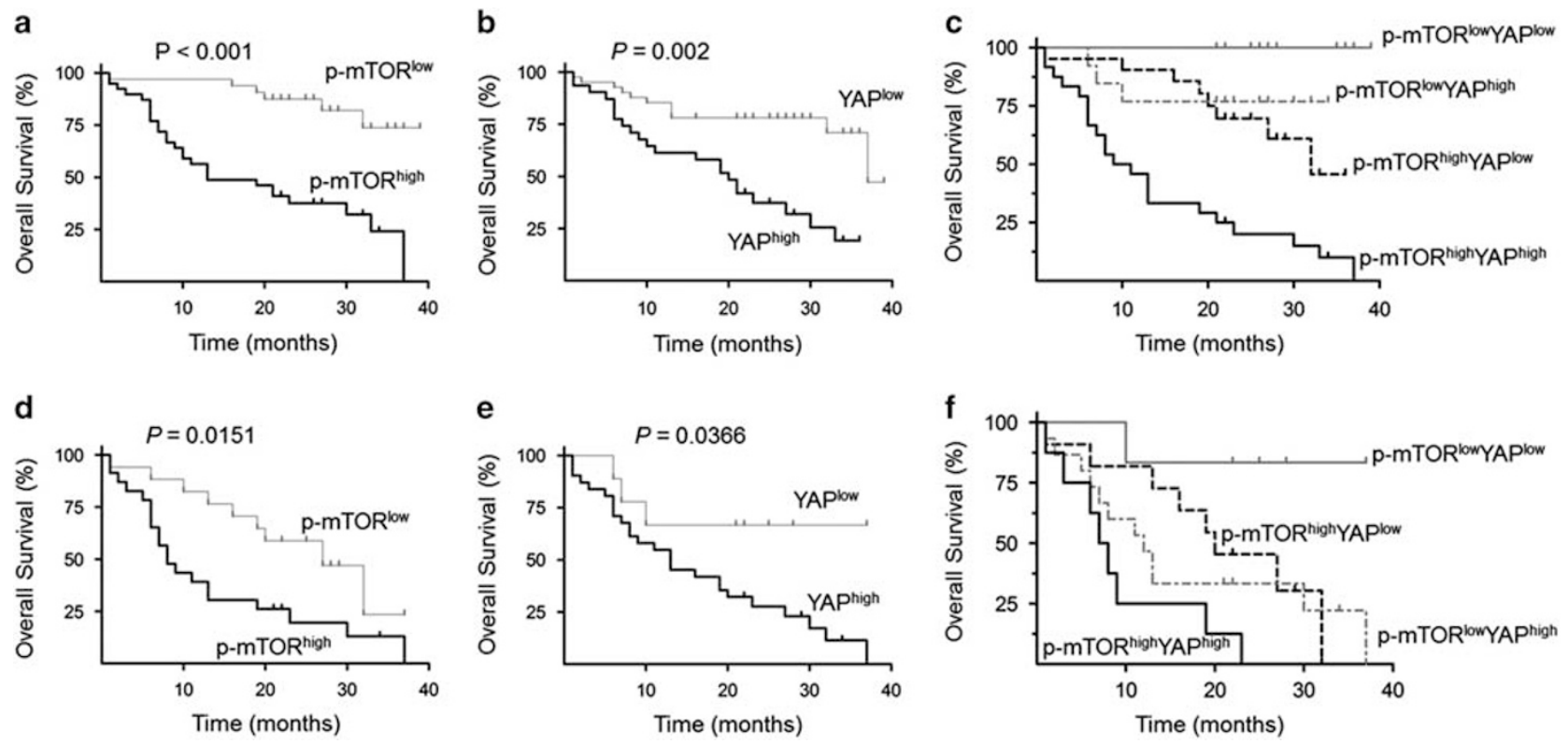

Figure 4 Kaplan-Meier survival analysis based on the expression of p-mTORS2448 and YAP. The relationship between p-mTOR expression and survival is calculated in all glioma tissues $(P<0.0001)$ (a) and in HGG $(P=0.0151)$ (d). The relationship of YAP expression and survival is calculated in all glioma tissues $(P=0.002)(\mathbf{b})$ and in HGG $(P=0.0366)(\mathbf{e})$. The relationship of $\mathrm{p}$-mTOR/YAP and survival is calculated in all glioma tissues

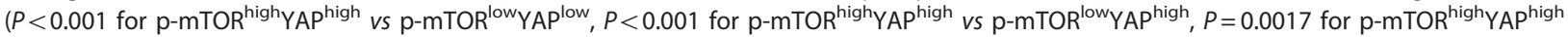
vs $\mathrm{p}$-mTORS2448 $\left.{ }^{\text {high }} Y A P^{\text {low }}\right)$ (c) and in HGG $\left(P=0.0019\right.$ for $p$-mTOR ${ }^{\text {high }} Y A P^{\text {high }}$ vs $p$-mTOR ${ }^{\text {low }} Y A P^{\text {low }}, P=0.0186$ for $p$-mTOR ${ }^{\text {high }} Y A P^{\text {high }}$ vs $\left.\mathrm{p}-\mathrm{mTOR}{ }^{\text {low }} Y \mathrm{P}^{\text {high }}\right)(\mathbf{f})$.

significantly higher in HGG (Figure 1), we next analyzed their prognostic significance specifically in HGG. Similar to our findings for all grades of gliomas, there was a significant inverse relationship between the length of overall survival and the expression of p-mTORS2448 ( $P=0.0151$; Figure $4 \mathrm{~d})$, YAP
$(P=0.0366$; Figure $4 \mathrm{e})$, as well as the combination of p-mTORS2448 and YAP $(P=0.0174$; Figure $4 \mathrm{f})$. The Cox regression analysis on $\mathrm{mTOR}, \mathrm{p}-\mathrm{mTOR}$, YAP, and $\mathrm{p}$-YAP further indicated that YAP protein levels can be an independent prognosis factor (Table 3). 
Evaluation of $m$ TOR and YAP as Therapeutic Targets in Glioma Cell Lines

To ascertain the biological activity of the crosstalk between the two signaling pathways, we examined the effect of
Everolimus (mTORC1 inhibitor) and peptide 17 (YAP inhibitor) alone or in combination on the growth of four glioma cell lines-CHG5 (grade II), SHG44 (grade III), T98G (grade IV), LN229 (grade IV) — and a normal glia—HEB by

Table 3 Cox regression analysis

\begin{tabular}{|c|c|c|c|c|c|c|c|c|}
\hline & \multicolumn{2}{|l|}{ mTOR } & \multicolumn{2}{|c|}{ p-mTORS2448 } & \multicolumn{2}{|l|}{ YAP } & \multicolumn{2}{|l|}{$p-Y A P$} \\
\hline & $\mathrm{HR}(95 \% \mathrm{Cl})$ & $P$-value & $\mathrm{HR}(95 \% \mathrm{Cl})$ & $P$-value & $\mathrm{HR}(95 \% \mathrm{Cl})$ & $P$-value & $\mathrm{HR}(95 \% \mathrm{Cl})$ & $P$-value \\
\hline All grades & $0.903(0.542-1.504)$ & 0.694 & $1.029(0.980-1.080)$ & 0.253 & 1.139 (1.074-1.207) & 0.000 & $0.944(0.846-1.043)$ & 0.244 \\
\hline $\mathrm{LGG}$ & $1.203(0.404-3.586)$ & 0.740 & $1.092(0.737-1.616)$ & 0.662 & $1.575(1.074-2.311)$ & 0.020 & $0.944(0.782-1.141)$ & 0.552 \\
\hline$H G G$ & $1.107(0.524-2.285)$ & 0.812 & $1.020(0.961-1.079)$ & 0.465 & $1.067(1.007-1.141)$ & 0.037 & $0.914(0.802-1.053)$ & 0.146 \\
\hline
\end{tabular}

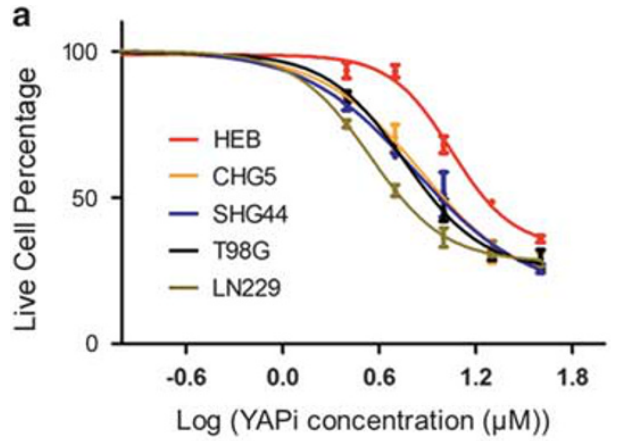

Log (YAPi concentration $(\mu \mathrm{M}))$
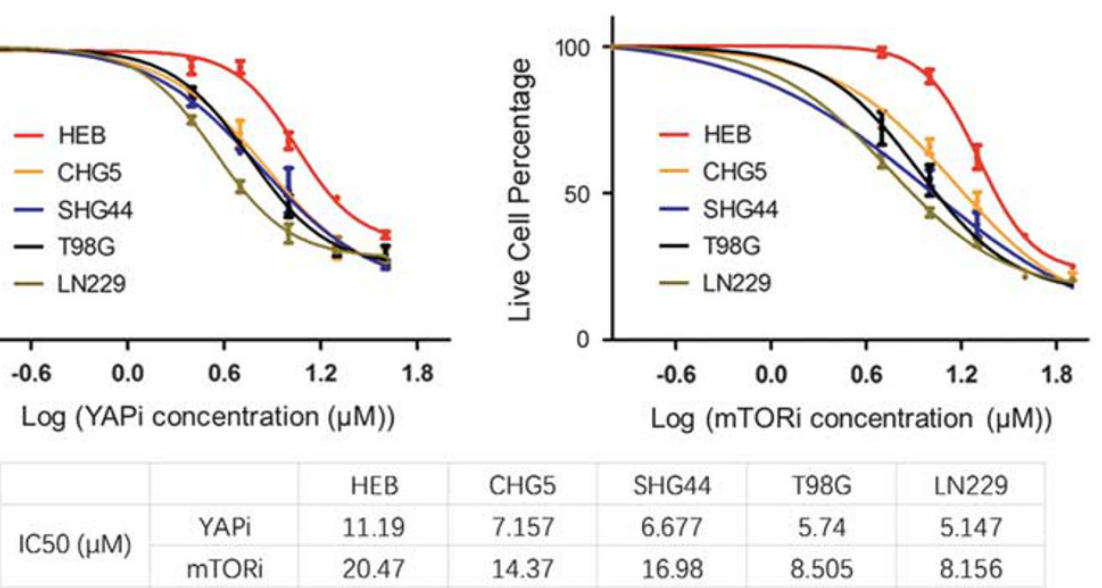

\begin{tabular}{|c|c|c|c|}
\hline CHG5 & SHG44 & T98G & LN229 \\
\hline 7.157 & 6.677 & 5.74 & 5.147 \\
\hline 14.37 & 16.98 & 8.505 & 8.156 \\
\hline
\end{tabular}
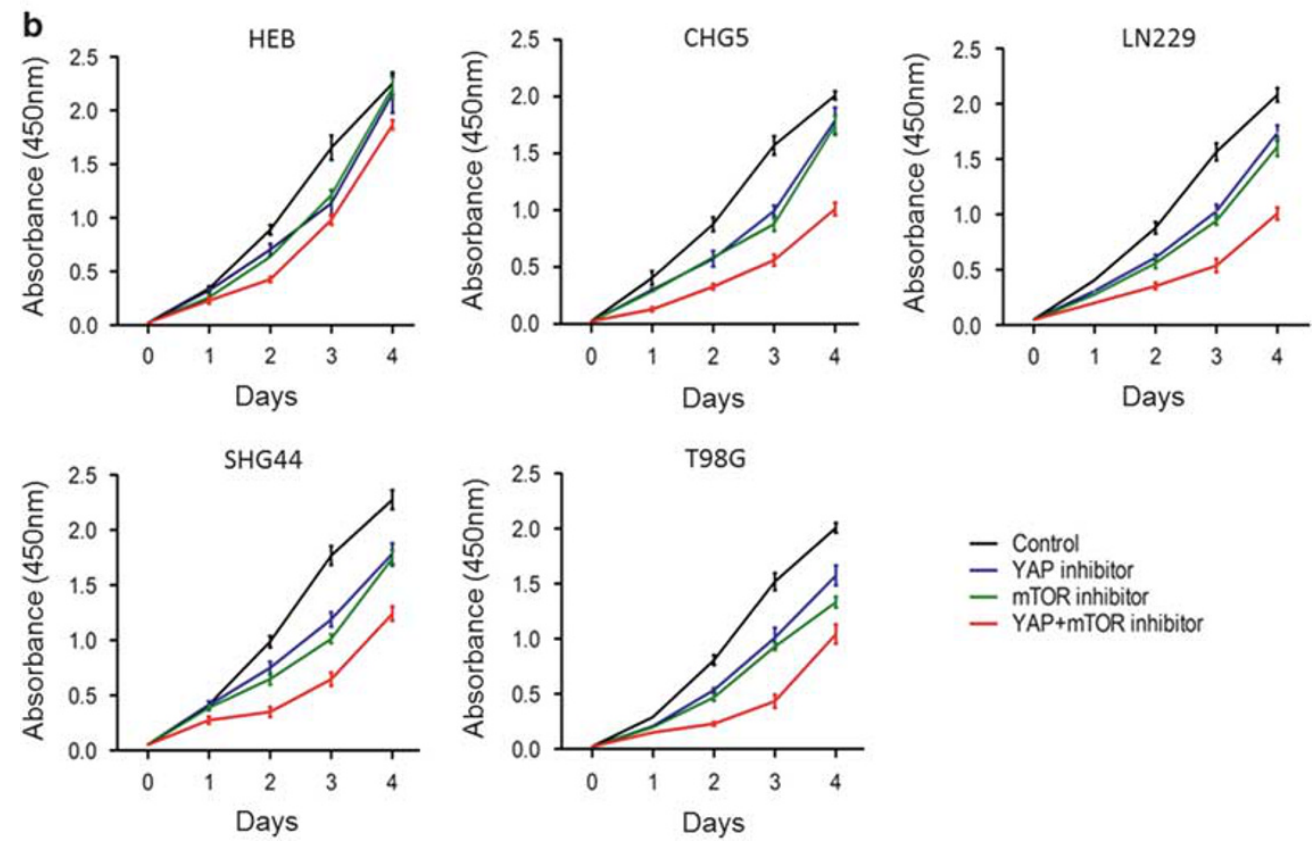

Figure 5 Growth assay of normal glia and glioma cell lines with treatments of mTOR inhibitor and YAP inhibitor. (a) IC50 of YAP inhibitor and mTOR inhibitor for the indicated cell lines. The data are means with s.d. $(n=3)$. (b) Cell viability assay of the indicated cell lines treated with mTORC1 inhibitor and YAP inhibitor alone or in combination. The data are means with s.d. $(n=3)$. 


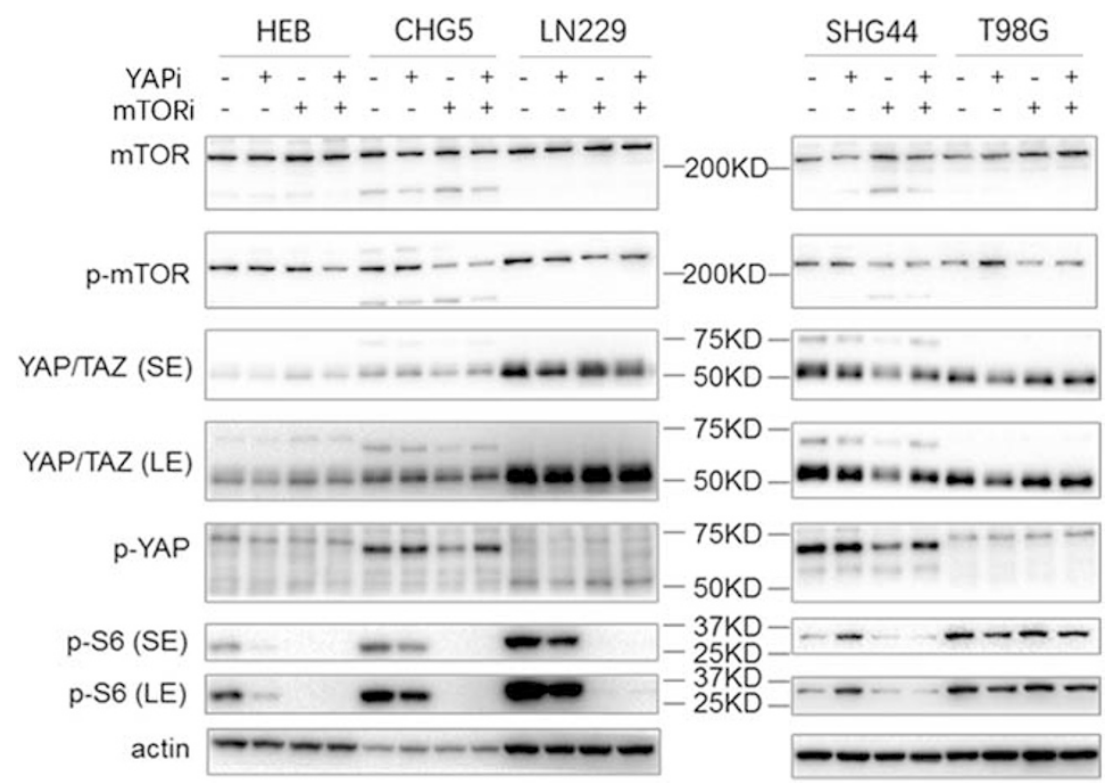

Figure 6 Western blotting analysis of normal glia and glioma cell lines with treatments of mTOR inhibitor and YAP inhibitor. LE, long exposure; $\mathrm{SE}$, short exposure.

CCK8 assay. We first examined the IC50 of the two inhibitors in these cell lines (Figure 5a), and the data showed that HEB cells had the highest IC50 values for both inhibitors among all five cell lines, meaning that HEB cell might be more resistant to the inhibitors than the other four glioma cell lines. The cell growth assay showed that the combination treatment using Everolimus and peptide 17 assumed more efficient inhibitory efficacy in the tumor cells compared with treatment with either Everolimus or peptide 17 alone, especially in LN229 cell line (Figure 5b). However, HEB cells were more resistant to the inhibitor treatments, either single or combination, than the glioma cell lines, which was also consistent with the highest IC50 of inhibitors for HEB cells (Figure 5a). Western blotting further showed that mTOR, p-mTORS2448, YAP, and p-YAP were clearly detected, and another mTORC1 marker, p-pS6 was also positive in all five cell lines, meaning that $\mathrm{mTOR}$ and YAP pathways were functionally involved in these cell lines (Figure 6). Interestingly, in LN229 cells, which possessed the comparable mTOR activation with other cell lines but highest YAP activation featured with the strongest total YAP and weak p-YAP signals (Figure 6), we observed the best combination effects (Figure $5 \mathrm{~b}$ ), which further supported our notion that co-examination of both mTOR phosphorylation and total YAP could be markers to predict combination effects (Figure 6).

\section{DISCUSSION}

In this work, we observed the positive correlation between protein levels of p-mTORS2448 and YAP in gliomas. Furthermore, the findings indicate that high expression levels of p-mTORS2448 and YAP correlate with poor overall survival of glioma patients. As p-mTORS2448 is a specific marker of
mTORC1 activation, our results implied an interaction between mTORC1 and YAP, which might functionally participate in the development and progress of gliomas. Although several mechanisms have been proposed for the crosstalk between the PI3K/AKT/mTORC1 and Hippo/YAP pathways, ${ }^{10,39,41-43}$ the clinical significance of the two pathways has not yet been assessed in a combinatorial manner. Our work, for the first time, reveals that p-mTORS2448 and YAP can be used as markers of the PI3K/AKT/mTORC1 and Hippo/YAP pathways to predict prognosis. It is worth mentioning that p-mTORS2448 and YAP also behaved as indicators of survival in HGG, and integration of the two markers could be applied to assess prognosis of patients with GBM, the most malignant form of glioma. ${ }^{1}$

The PI3K/AKT/mTOR axis induces phosphorylation of mTOR at Ser2448 followed by the activation of mTORC1, 32,33 and thus targeting mTORC1 by rapamycin and its analogs (rapalogs) is a potent way to block this oncogenic axis, which have been extensively examined in clinical trials. Several rapalogs have been developed by pharmaceutical companies to target mTORC1 in organ transplantation and cancer treatment, such as rapamycin (Sirolimus), Temsirolimus, Everolimus, and Deforolimus. ${ }^{29,30,36}$ Everolimus has been approved to treat subependymal giant cell astrocytoma, a type of grade I glioma. ${ }^{51}$ A phase II trial of Everolimus, temozolomide, and radiotherapy in patients with GBM suggested that, in spite of an initial antiproliferative effect in a genetically distinct subset of tumors, the treatment did not increase survival compared with conventional therapy, ${ }^{52}$ suggesting that other markers may be required. In addition, Temsirolimus has not shown satisfactory effects on GBM in phase II clinical trial due to insufficient inhibition of mTOR 
pathway. ${ }^{53,54}$ Similarly, it has been reported that PI3K-mTOR pathway inhibition showed modest efficacy on HGG when used as a single agent, and it may be more effective to use it in combination with inhibitors targeting other activated pathways. ${ }^{38}$ These results indicate that combination of an mTOR inhibitor with another agent may be more beneficial than application of an individual mTOR inhibitor. In support of this concept, combination treatment has been reported in several preclinical or clinical trials for multiple cancer types, including gliomas. For example, Erlotinib (EGFR inhibitor) and Temsirolimus have been used to treat HGG in phase I/II clinical trials. ${ }^{55}$ The combination of Everolimus, temozolomide, and radiation therapy has been tested in patients with newly diagnosed GBM in a phase I clinical trial. ${ }^{56}$ Temsirolimus has also been tested in combination with chemoradiation or bevacizumab for GBM. ${ }^{57,58}$ Therefore, combination treatment including an mTORC1 inhibitor might be a useful strategy for glioma with mTORC1 overexpression. So far, the YAP inhibitor has not yet been tested in clinical trials for GBM, but our work herein suggests that the YAP inhibitor may enhance the effects of mTOR inhibitors, which provides a new avenue for the combination treatment. Interestingly, in LN229 cells, which showed the highest levels of YAP activation and high total YAP with low phosphorylated YAP, we observed the best combination effects, which further supports our notion that examination of both mTOR phosphorylation and total YAP are markers to predict the efficacy of combination treatment.

Although the mechanisms on the crosstalk of mTOR and Hippo/YAP pathways have not been further explored, our data herein, through specimen-based analysis and in vitro assays, indicate that the protein levels of p-mTORS2448 and YAP not only serve as markers to predict patient prognosis but also as therapeutic markers to stratify patients for target therapy with mTOR and YAP inhibitors.

\section{ACKNOWLEDGMENTS}

This work was supported by the Thousand Talents Plan - Youth Program to YW, the National Key Research and Development Program of China (No. 2016YFA0101203) to XWB, the National Natural Science Foundation of China (No. 81230062 and No. 61327902-4) to XWB, (No. 81402066) to YLY, (No. 81602197) to JT.

\section{DISCLOSURE/CONFLICT OF INTEREST}

The authors declare no conflict of interest.

1. Crespo I, Vital AL, Gonzalez-Tablas $\mathrm{M}$, et al. Molecular and genomic alterations in glioblastoma multiforme. Am J Pathol 2015;185: 1820-1833.

2. Louis DN, Perry A, Reifenberger G, et al. The 2016 World Health Organization Classification of Tumors of the Central Nervous System: a summary. Acta Neuropathol 2016;131:803-820.

3. Ostrom QT, Gittleman H, de Blank PM, et al. American Brain Tumor Association Adolescent and Young Adult Primary Brain and Central Nervous System Tumors Diagnosed in the United States in 2008-2012. Neuro Oncol 2016;18(Suppl 1):i1-i50.

4. Tanaka S, Louis DN, Curry WT, et al. Diagnostic and therapeutic avenues for glioblastoma: no longer a dead end? Nat Rev Clin Oncol 2013;10:14-26.
5. Rodriguez FJ, Vizcaino MA, Lin MT. Recent advances on the molecular pathology of glial neoplasms in children and adults. J Mol Diagn 2016;18:620-634.

6. Chen R, Cohen AL, Colman H. Targeted therapeutics in patients with high-grade gliomas: past, present, and future. Curr Treat Options Oncol 2016;17:42.

7. Liang N, Pende M. YAP enters the mTOR pathway to promote tuberous sclerosis complex. Mol Cell Oncol 2015;2:e998100.

8. Shimobayashi M, Hall MN. Making new contacts: the mTOR network in metabolism and signalling crosstalk. Nat Rev Mol Cell Biol 2014;15: 155-162.

9. Csibi A, Blenis J. Hippo-YAP and mTOR pathways collaborate to regulate organ size. Nat Cell Biol 2012;14:1244-1245.

10. Tumaneng K, Schlegelmilch $\mathrm{K}$, Russell RC, et al. YAP mediates crosstalk between the Hippo and PI(3)K-TOR pathways by suppressing PTEN via miR-29. Nat Cell Biol 2012;14:1322-1329.

11. Artinian N, Cloninger C, Holmes B, et al. Phosphorylation of the hippo pathway component AMOTL2 by the mTORC2 kinase promotes YAP signaling, resulting in enhanced glioblastoma growth and invasiveness. J Biom Chem 2015;290:19387-19401.

12. Chao $Y$, Wang $Y$, Liu $X$, et al. Mst1 regulates glioma cell proliferation via the AKT/mTOR signaling pathway. J Neurooncol 2015;121:279-288.

13. Justice RW, Zilian O, Woods DF, et al. The Drosophila tumor suppressor gene warts encodes a homolog of human myotonic dystrophy kinase and is required for the control of cell shape and proliferation. Genes Dev 1995;9:534-546.

14. Xu T, Wang W, Zhang $S$, et al. Identifying tumor suppressors in genetic mosaics: the Drosophila lats gene encodes a putative protein kinase. Development 1995;121:1053-1063.

15. Meng Z, Moroishi T, Guan KL. Mechanisms of Hippo pathway regulation. Genes Dev 2016;30:1-17.

16. Plouffe SW, Meng Z, Lin KC, et al. Characterization of Hippo pathway components by gene inactivation. Mol Cell 2016;64:993-1008.

17. Yu FX, Zhao B, Guan KL. Hippo pathway in organ size control, tissue homeostasis, and cancer. Cell 2015;163:811-828.

18. Halder G, Johnson RL. Hippo signaling: growth control and beyond Development 2011;138:9-22.

19. Pan D. The hippo signaling pathway in development and cancer. Dev Cell 2010;19:491-505.

20. Yu FX, Guan KL. The Hippo pathway: regulators and regulations. Genes Dev 2013;27:355-371.

21. Lian I, Kim J, Okazawa $\mathrm{H}$, et al. The role of YAP transcription coactivator in regulating stem cell self-renewal and differentiation. Genes Dev 2010;24:1106-1118.

22. Zanconato F, Forcato M, Battilana G, et al. Genome-wide association between YAP/TAZ/TEAD and AP-1 at enhancers drives oncogenic growth. Nat Cell Biol 2015;17:1218-1227.

23. Fernandez LA, Squatrito $M$, Northcott $P$, et al. Oncogenic YAP promotes radioresistance and genomic instability in medulloblastoma through IGF2-mediated Akt activation. Oncogene 2012;31:1923-1937.

24. Chan SW, Lim CJ, Guo K, et al. A role for TAZ in migration, invasion, and tumorigenesis of breast cancer cells. Cancer Res 2008;68:2592-2598.

25. Moroishi T, Hansen CG, Guan KL. The emerging roles of YAP and TAZ in cancer. Nat Rev Cancer 2015;15:73-79.

26. Steinhardt AA, Gayyed MF, Klein AP, et al. Expression of Yes-associated protein in common solid tumors. Hum Pathol 2008;39:1582-1589.

27. Zhou Z, Hu T, Xu Z, et al. Targeting Hippo pathway by specific interruption of YAP-TEAD interaction using cyclic YAP-like peptides. FASEB J 2015;29:724-732.

28. Zhang Z, Lin Z, Zhou Z, et al. Structure-based design and synthesis of potent cyclic peptides inhibiting the YAP-TEAD protein-protein interaction. ACS Med Chem Lett 2014;5:993-998.

29. Laplante M, Sabatini DM. mTOR signaling in growth control and disease. Cell 2012;149:274-293.

30. Guertin DA, Sabatini DM. Defining the role of mTOR in cancer. Cancer Cell 2007;12:9-22.

31. Nathan N, Keppler-Noreuil KM, Biesecker LG, et al. Mosaic disorders of the PI3K/PTEN/AKT/TSC/mTORC1 signaling pathway. Dermatol Clin 2017;35:51-60.

32. Huang $\mathrm{S}$, Yang ZJ, Yu C, et al. Inhibition of mTOR kinase by AZD8055 can antagonize chemotherapy-induced cell death through autophagy induction and down-regulation of p62/sequestosome 1. J Biol Chem 2011;286:40002-40012. 
33. Nave BT, Ouwens $\mathrm{M}$, Withers DJ, et al. Mammalian target of rapamycin is a direct target for protein kinase B: identification of a convergence point for opposing effects of insulin and amino-acid deficiency on protein translation. Biochem J 1999;344(Pt 2):427-431.

34. Ma XM, Blenis J. Molecular mechanisms of mTOR-mediated translational control. Nat Rev Mol Cell Biol 2009;10:307-318.

35. Perl A. mTOR activation is a biomarker and a central pathway to autoimmune disorders, cancer, obesity, and aging. Ann NY Acad Sci 2015;1346:33-44.

36. Zoncu R, Efeyan A, Sabatini DM. mTOR: from growth signal integration to cancer, diabetes and ageing. Nat Rev Mol Cell Biol 2011;12:21-35.

37. Li X, Wu C, Chen $\mathrm{N}$, et al. PI3K/Akt/mTOR signaling pathway and targeted therapy for glioblastoma. Oncotarget 2016;7:33440-33450.

38. Lin F, de Gooijer MC, Hanekamp D, et al. PI3K-mTOR pathway inhibition exhibits efficacy against high-grade glioma in clinically relevant mouse models. Clin Cancer Res 2016;23:1286-1298.

39. Hansen CG, Ng YL, Lam WL, et al. The Hippo pathway effectors YAP and TAZ promote cell growth by modulating amino acid signaling to mTORC1. Cell Res 2015;25:1299-1313.

40. Nelson N, Clark GJ. Rheb may complex with RASSF1A to coordinate Hippo and TOR signaling. Oncotarget 2016;7:33821-33831.

41. Dill PE, Liang N, Pende $M$. New insights into the pathophysiology of the tuberous sclerosis complex: crosstalk of mTOR- and hippo-YAP pathways in cell growth. Rare Dis 2015;3:e1016701.

42. Liang N, Zhang C, Dill P, et al. Regulation of YAP by mTOR and autophagy reveals a therapeutic target of tuberous sclerosis complex. J Exp Med 2014;211:2249-2263.

43. Park YY, Sohn BH, Johnson RL, et al. Yes-associated protein 1 and transcriptional coactivator with PDZ-binding motif activate the mammalian target of rapamycin complex 1 pathway by regulating amino acid transporters in hepatocellular carcinoma. Hepatology 2016;63:159-172.

44. Ping YF, Yao XH, Jiang JY, et al. The chemokine $\mathrm{CXCL12}$ and its receptor CXCR4 promote glioma stem cell-mediated VEGF production and tumour angiogenesis via PI3K/AKT signalling. J Pathol 2011;224: 344-354.

45. Yang $\mathrm{SX}, \mathrm{Chen} \mathrm{JH}$, Jiang $\mathrm{XF}$, et al. Activation of chemokine receptor CXCR4 in malignant glioma cells promotes the production of vascular endothelial growth factor. Biochem Biophys Res Commun 2005;335: 523-528.
46. Rao J, Zhou ZH, Yang J, et al. Semaphorin-3F suppresses the stemness of colorectal cancer cells by inactivating Rac1. Cancer Lett 2015;358: 76-84.

47. Sharma S, Sharma MC, Gupta DK, et al. Angiogenic patterns and their quantitation in high grade astrocytic tumors. J Neurooncol 2006;79: 19-30.

48. Sedrani R, Cottens $S$, Kallen J, et al. Chemical modification of rapamycin: the discovery of SDZ RAD. Transplant Proc 1998;30:2192-2194.

49. Shi Y, Chen C, Yu SZ, et al. miR-663 suppresses oncogenic function of CXCR4 in glioblastoma. Clin Cancer Res 2015;21:4004-4013.

50. Verhaak RG. Moving the needle: optimizing classification for glioma. Sci Transl Med 2016;8:350fs314.

51. Sasongko TH, Ismail NF, Zabidi-Hussin Z. Rapamycin and rapalogs for tuberous sclerosis complex. Cochrane Database Syst Rev 2016;7: CD011272.

52. Ma DJ, Galanis E, Anderson SK, et al. A phase II trial of everolimus, temozolomide, and radiotherapy in patients with newly diagnosed glioblastoma: NCCTG N057K. Neuro Oncol 2015;17:1261-1269.

53. Chang SM, Wen P, Cloughesy T, et al. Phase II study of CCl-779 in patients with recurrent glioblastoma multiforme. Invest New Drugs 2005:23:357-361.

54. Galanis E, Buckner JC, Maurer MJ, et al. Phase II trial of temsirolimus (CCl-779) in recurrent glioblastoma multiforme: a North Central Cancer Treatment Group Study. J Clin Oncol 2005;23:5294-5304.

55. Wen PY, Chang SM, Lamborn KR, et al. Phase I/II study of erlotinib and temsirolimus for patients with recurrent malignant gliomas: North American Brain Tumor Consortium trial 04-02. Neuro Oncol 2014;16: 567-578.

56. Sarkaria JN, Galanis E, Wu W, et al. North Central Cancer Treatment Group Phase I trial N057K of everolimus (RAD001) and temozolomide in combination with radiation therapy in patients with newly diagnosed glioblastoma multiforme. Int J Radiat Oncol Biol Phys 2011;81:468-475.

57. Sarkaria JN, Galanis E, Wu W, et al. Combination of temsirolimus (CCl-779) with chemoradiation in newly diagnosed glioblastoma multiforme (GBM) (NCCTG trial N027D) is associated with increased infectious risks. Clin Cancer Res 2010;16:5573-5580.

58. Lassen U, Sorensen M, Gaziel TB, et al. Phase II study of bevacizumab and temsirolimus combination therapy for recurrent glioblastoma multiforme. Anticancer Res 2013;33:1657-1660. 